Revue d'histoire de l'Amérique française

REYUE D.HISTOIRE DE L'AMÉRIQUE FRANÇAISE

\title{
CALOREN, Fred, HARVEY, Julien, JULIEN, Claude, NAUD, André, RIOUX, Bertrand, ROCHER, Guy, RYAN, Claude, THUR, Livia, Le nouveau défi des valeurs - Essais. Collection Constantes 20. Les Éditions HMH, Montréal, 1969. 172 p. \$2.50.
}

\section{Edmond Orban}

Volume 23, numéro 2, septembre 1969

URI : https://id.erudit.org/iderudit/302888ar

DOI : https://doi.org/10.7202/302888ar

Aller au sommaire du numéro

Éditeur(s)

Institut d'histoire de l'Amérique française

ISSN

0035-2357 (imprimé)

1492-1383 (numérique)

Découvrir la revue

Citer ce compte rendu

Orban, E. (1969). Compte rendu de [CALOREN, Fred, HARVEY, Julien, JULIEN, Claude, NAUD, André, RIOUX, Bertrand, ROCHER, Guy, RYAN, Claude, THUR, Livia, Le nouveau défi des valeurs - Essais. Collection Constantes 20. Les Éditions HMH, Montréal, 1969. 172 p. \$2.50.] Revue d'histoire de l'Amérique française, 23(2), 317-319. https://doi.org/10.7202/302888ar d'utilisation que vous pouvez consulter en ligne. 
Caloren, Fred, Harvey, Julien, Julien, Claude, Naud, André, RIOUX, Bertrand, Rocher, Guy, RYAN, Claude, THUR, Livia, Le nouveau défi des valeurs - Essais. Collection Constantes 20. Les Editions HMH, Montréal, 1969. 172 pages. $\$ 2.50$.

Ce recueil de textes présente un intérêt certain, à cause de l'actualité du sujet traité et aussi en raison de la clarté et de la concision qui caractérisent ces diverses interventions. Quant aux auteurs et commentateurs, ils appartiennent à des disciplines très variées qui souvent se recoupent d'ailleurs, mais au sein desquelles les sciences sociales et la philosophie dominent nettement. A cet égard on regrettera avec l'éditeur que le texte du docteur Karl Stern n'ait pu être publié. Il aurait avantageusement complété cet éventail.

Soulignons au départ que ces personnes ont au moins un commun dénominateur de base en ce sens qu'elles semblent admettre un certain nombre de valeurs que le christianisme, pris dans une perspective globale et 
idéale, accepterait comme siennes. Ceci, à première vue, peut restreindre le champ de discussion. On aurait aimé entendre des personnes venant d'autres horizons, mais la formule utilisée a au moins l'avantage de faire de ce tout un ensemble logique et cohérent, précisément parce qu'on part de prémisses communes. D'ailleurs, peu importent les antécédents des participants, c'est ce qu'ils disent qui nous intéresse avant tout.

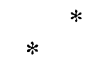

S'attachant plus particulièrement au Québec le sociologue Guy Rocher y analyse la crise des valeurs. Il utilise même les termes angoisse, panique, pour caractériser ce passage sans transition de la société industrielle à la société post-industrielle. Les transformations profondes et rapides du milieu social provoquent à leur tour une rupture entre les valeurs dites traditionnelles et les valeurs nouvelles. Ces valeurs "nouvelles" recherchées par la jeunesse pourraient s'appeler : fraternité humaine, égalité, amour, efficience, compétence, etc. Le passé a changé de signification et la religion, dans beaucoup de ses manifestations extérieures, semble encore se rattacher à des valeurs jugées du type traditionnel. Mais, se demande Guy Rocher, existe-t-il tellement d'écart entre les véritables valeurs chrétiennes et les valeurs nouvelles. Il y a certes beaucoup d'ambiguïté et de malentendu à ce sujet. D'autre part l'attitude contradictoire de beaucoup de chrétiens est responsable en partie de cet état de choses. Et ici on reprend un thème cher à Bernanos, celui qu'en des termes plus virulents ce dernier aurait appelé: la trahison, la prostitution des valeurs chrétiennes. La démonstration faite est susceptible d'émouvoir ceux qui ne sont pas trop sceptiques.

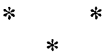

Quant à Claude Julien, rédacteur au journal Le Monde, il traite d'une façon un peu impromptue la question de la faillite des idéologies. Les Soviétiques, écrit-il, tendent à rejoindre les Américains dans leur souci d'efficacité dans la production et leur désir forcené d'une société d'abondance et de haute consommation. Ce serait la convergence de deux types de matérialisme. Critiquant les pays communistes, il souligne qu'eux aussi ont trahi leur idéal premier, l'homme nouveau communiste y est mort-né, c'est le profit personnel qui tend à l'emporter à travers un nationalisme égoïste. Quant aux libertés, la stratocratie les foule aux pieds. Repassant de l'autre côté de la barricade, Claude Julien s'en prend cette fois aux EtatsUnis. La révolution américaine réalisée au nom de l'égalité et de la liberté a dévié, on le constate, sur le plan interne avec les zones de pauvreté et davantage encore dans les rapports qu'entretiennent les Etats-Unis avec le Tiers Monde. Il s'ensuit une démonstration à partir de faits d'actualité que nous connaissons déjà suffisamment (Par souci de rétablir l'équilibre rompu par les superpuissances on risque parfois d'être un peu subjectif, ne fut-ce qu'en ne voyant qu'un côté de la médaille).

Finalement se pose la question par laquelle on aurait dû commencer, quelles sont les valeurs essentielles ? Pour Claude Julien, ce sont: la vérité, la liberté, la justice, la beauté, l'amour. Or il n'y a pas de place pour ces 
valeurs dans une société fondée trop exclusivement sur la notion de profit matériel et d'abondance de biens de consommation. Quant au christianisme, s'il veut survivre, il doit revenir concrètement à ces valeurs qu'après tout il a contribué à engendrer.

L'exposé de Bertrand Rioux aurait gagné à figurer au début de ce livre. Il définit le concept valeur, insiste sur son ambiguïté. Il y a des valeurs nouvelles, mais peut-être aussi des valeurs constantes. Il examine sommairement les rapports pouvant exister entre la valeur et l'essence de la liberté. Finalement il semble accepter la possibilité qu'il y ait un fondement transcendant aux valeurs. "Dire que les valeurs sont transcendantes, c'est dire que nos actes qui les incarnent, ne les égalent jamais." L'autre aspect de la transcendance des valeurs, c'est celui d'une visée absolue impliquée dans la valeur.

L'exposé de Fred Caloren est très intéressant en soi, mais venant après les autres, il ne fait que confirmer et compléter. Il traite de la "déshumanisation" qu'apporte un monde technique encore mal organisé, de l'efficacité aux dépens de la qualité de la vie. La contestation ne serait qu'une forme de révolte désespérée contre une société décadente. Malgré les apparences contraires souvent, on peut se demander si au fond tous ces jeunes, à quelque degré que ce soit, ne recherchent pas les valeurs fondamentales mentionnées plus haut. Il ne reste guère de place pour mentionner l'exposé de Julien Harvey sur l'assomption chrétienne des nouvelles valeurs. Comme on pouvait s'y attendre de la part d'un théologien, Dieu est le fondement ultime de toute valeur digne de ce nom. La démonstration est bien étayée, mais certains préféreront d'autres approches.

On reste cependant étonné de voir comment tous ces participants débouchent sur la conclusion qu'à côté de valeurs relatives, il y a des valeurs permanentes. Mais pour cela, comme l'écrivait Franz Oppenheimer, dans L'Etat, "il faut reculer assez loin, placer les points d'observation assez haut, pour que le jeu bigarré des détails ne nous cache pas les grands mouvements de masse". Concluons en constatant que tous les participants à ces essais semblent avoir gardé un minimum d'espérance, de quoi peut-être surmonter la tension des valeurs et partant d'éviter une capitulation définitive puisqu'ils croient à des valeurs permanentes. 